\title{
Risk factors and long-term outcomes of elderly patients complicating with acute kidney injury after type $A$ acute aortic dissection surgery: a retrospective study
}

\author{
Zhigang Wang, Min Ge, Tao Chen, Cheng Chen, Qiuyan Zong, Lichong Lu, Kunsheng Li, Dongjin Wang \\ Department of Cardio-thoracic Surgery, Nanjing Drum Tower Hospital, The Affiliated Hospital of Nanjing University Medical School, Nanjing, \\ China \\ Contributions: (I) Conception and design: D Wang; (II) Administrative support: D Wang, M Ge; (III) Provision of study materials or patients: T Chen, \\ C Chen, Q Zong; (IV) Collection and assembly of data: Z Wang, K Li, L Lu; (V) Data analysis and interpretation: Z Wang, M Ge; (VI) Manuscript \\ writing: All authors; (VII) Final approval of manuscript: All authors. \\ Correspondence to: Dongjin Wang. Department of Cardio-thoracic Surgery, Nanjing Drum Tower Hospital, The Affiliated Hospital of Nanjing \\ University Medical School, Nanjing 210008, China. Email: glyywdj@163.com.
}

\begin{abstract}
Background: To identify risk factors and long-term outcomes for acute kidney injury (AKI) in elderly patients who underwent type A acute aortic dissection (TA-AAD) emergency surgeries.

Methods: This retrospective study enrolled 214 consecutive patients who underwent TA-AAD emergency surgeries between January 2014 to December 2018 in Nanjing Drum Tower hospital. The diagnosis of AKI was made based on the Kidney Disease: Improving Global Outcomes definition (KDIGO) criteria. Multivariable regression analysis was performed to identify risk factors for postoperative AKI. KaplanMeier curves were generated to compare the long-term outcomes between patients with and without AKI complication after TA-AAD surgeries.

Results: Among all enrolled patients, 114 (53.3\%) developed AKI during postoperative period. The median age of patients with or without AKI was $68.0(64.0,74.0)$ and $66.0(62.0,72.8)$ years respectively. Renal replacement therapy (RRT) was required in 43 patients $(20.1 \%)$. The 30 -day mortality rate was $21.5 \%$ in all enrolled patients with $26.3 \%$ in AKI group and $16.0 \%$ in non-AKI group $(\mathrm{P}=0.067)$ respectively. Longer mechanical ventilation duration was identified as the only independent risk factor for developing AKI by multivariable logistic regression analysis. In addition, our data suggested that the long-term cumulative survival rate was different between two groups.

Conclusions: Postoperative AKI after TA-AAD surgeries was common and associated with worsened long-term mortality in elderly patients. Longer postoperative mechanical ventilation duration was identified as the only independent risk factor for the development of AKI.
\end{abstract}

Keywords: Acute kidney injury (AKI); type A aortic dissection; risk factors; elderly; mechanical ventilation

Submitted May 24, 2020. Accepted for publication Aug 21, 2020.

doi: $10.21037 /$ jtd-20-2018

View this article at: http://dx.doi.org/10.21037/jtd-20-2018

\section{Introduction}

Acute kidney injury (AKI) is a common complication after aortic dissection surgery that increases patients' mortality rate (1). The incidence of AKI after aortic surgery was reported from $20 \%$ to $67 \%$, which was higher compared with other cardiac procedures (2). It has been known that the development of AKI after type A acute aortic dissection (TA$\mathrm{AAD}$ ) surgeries also decreases long-term survival rate (3).

In our aging society, the number of elderly patients undergoing emergency surgery for TA-AAD has been steadily increasing. Epidemiology studies have shown that people with advancing age are associated with increasing 
Table 1 Kidney Disease Improving Global Outcomes (KDIGO) criteria for acute kidney injury

\begin{tabular}{ll}
\hline Stage & Serum creatinine $(\mathrm{sCr})$ increase \\
\hline 1 & $1.5-1.9$ times baseline or $\geq 0.3 \mathrm{mg} / \mathrm{dL}$ increase \\
2 & $2.0-2.9$ times baseline \\
3 & $>3.0$ times baseline or increase in $\mathrm{sCr}$ to $\geq 4.0 \mathrm{mg} / \mathrm{dL}$ \\
& or initiation of renal replacement therapy \\
\hline
\end{tabular}

possibility of developing AKI, possibly due to the intrinsic morphological and functional changes of their kidneys (4). In addition, other factors such as preexisting comorbidities and overall declined health conditions may also contribute to AKI development. However, the association between AKI and TA-AAD surgeries in elderly has not been well studied. In order to identify patients with increased risk, this retrospective study was designed to investigate the incidence, risk factors and long-term outcomes of AKI in elderly patients who underwent emergency surgery for TA$\mathrm{AAD}$. We present the following article in accordance with the STROBE reporting checklist (available at http://dx.doi. org/10.21037/jtd-20-2018).

\section{Methods}

\section{Patients}

The ethics committee of Nanjing Drum Tower hospital approved this retrospective study and waived the need for individual informed consent due to the nature of this study. The study was conducted in accordance with the Declaration of Helsinki (as revised in 2013). 222 consecutive patients who enrolled in this study were all above 60 years old and underwent emergent TA-AAD surgery between January 2014 to December 2018 in Nanjing Drum Tower hospital. Their medical records were reviewed retrospectively. Patients who received preoperative renal replacement therapy (RRT) were excluded because of the difficulty in measuring the progression of renal dysfunction. Patients who died within 24 hours postoperatively were excluded were also excluded because their mortality was not associated with renal dysfunction. Eventually, a total of 214 patients enrolled in this study.

\section{Definitions}

Elderly was defined according to Chinese law (>60 years old). The diagnosis of AKI was made according to the Kidney Disease Improving Global Outcomes (KDIGO) criteria which mainly based on the increase of serum creatine $(\mathrm{sCr})(5)$. The diagnosing criteria and classification were shown in Table 1. The diagnosis of aortic dissection was verified in all patients with computed tomography angiography (CTA) examination of aorta. The acute and subacute phases were defined as the time from the symptom onset to hospital admission less than 14 days. The posterior parallel circulation (PPC) time referred to the time from opening of ascending aorta to completion of cardiopulmonary bypass (CPB). The kidney blood supply was divided into true lumen, false lumen or double cavity blood supply groups based on preoperative CTA.

\section{Outcomes}

The primary endpoint was long-term survival rate. The events associated with primary endpoint referred to death from any cause. Evaluation of patients' general health status was conducted through questionnaire once a year to all patients who have undergone TA-AAD operation since 2014. If patients did not respond to mailed questionnaire, telephone contact was made to obtain current health status information. If patients passed away at the time of telephone contact, information about date and cause of death was obtained from relatives. Cause of death were categorized as follows: aortic rupture, cardiac failure, respiratory failure and others. The median follow-up period was 29 months.

Secondary endpoints were early postoperative clinical characteristics and surgical complications. The early postoperative clinical characteristics included drainage volume 24 hours after surgery, mechanical ventilation duration, 30-day mortality, intensive care unit (ICU) stay time and hospital stay time. Surgical complications included re-exploration for bleeding, postoperative renal replacement therapy (RRT), stroke, paraplegia, tracheostomy and deep sternal wound infection (Table 2).

\section{Strategies to prevent postoperative AKI}

Prompt resuscitation of the circulation with fluids, vasopressors and inotropes remains the cornerstone in prevention of AKI. Volume expansion with isotonic crystalloids was only recommended when hypovolemia was suspected. Following or together with fluid resuscitation hypotensive patients were given vasoconstrictor, preferably norepinephrine. All drugs known to be nephrotoxic were 
Table 2 Comparison of postoperative variables

\begin{tabular}{|c|c|c|c|}
\hline Variables & AKI $(n=114)$ & Non-AKI $(n=100)$ & $P$ value ${ }^{a}$ \\
\hline Drainage volume 24 hours after surgery $(\mathrm{mL})$ & $525.0(327.5,972.5)$ & $450.0(255.5,690.0)$ & 0.155 \\
\hline Ventilation time (hour) & $25.5(12.8,65.5)$ & $14.0(10.0,21.6)$ & 0.002 \\
\hline 30-day mortality (\%) & $30(26.3)$ & $16(16.0)$ & 0.067 \\
\hline Hospital stay time (day) & $21.5(15.0,32.3)$ & $18.0(14.0,23.0)$ & 0.006 \\
\hline \multicolumn{4}{|l|}{ Surgical complications } \\
\hline Re-exploration for bleeding (\%) & $5(4.4)$ & $5(5.0)$ & 1.000 \\
\hline Dialysis (\%) & $31(27.2)$ & $12(12.0)$ & 0.006 \\
\hline Deep sternal wound infection (\%) & $2(1.8)$ & $3(3.0)$ & 0.666 \\
\hline
\end{tabular}

Data presented as $\mathrm{n}(\%)$; median (IQR). ICU, intensive care unit. ${ }^{\mathrm{a}} \mathrm{P}$ values indicate differences between $\mathrm{AKI}$ and $\mathrm{Non}-\mathrm{AKI}$. $\mathrm{P}<0.05$ was considered statistically significant.

used with extreme cautious and discontinued promptly when possible. Diuretics should not be used for prevention of AKI alone but may benefit renal function by relieving renal congestion (6). When elevated sCr was discovered postinitial surgery, medications such as ketosteril, compound amino acid, or calcium dobesilate were prescribed.

\section{Surgical procedures}

All patients underwent standard median sternotomy and total CPB. After systemic heparinization, $\mathrm{CPB}$ was established by cannulation of femoral artery or right axillary artery with right atrium. Cannulation of the right axillary artery was preferred when $\mathrm{CPB}$ and unilateral selective cerebral perfusion (SCP) were both required. The mean arterial pressure was maintained between 55 and $75 \mathrm{mmHg}$. After the establishment of $\mathrm{CPB}$, systemic heparinization was initiated. Next, after clamping the ascending aorta, cardiac arrest was accomplished with cold cardioplegic solution, and cold blood cardioplegia (4:1 blood:crystalloid ratio) was infused by both anterograde and retrograde infusion method. Circulation arrest was initiated when cooling reached its target rectal temperature of $22^{\circ} \mathrm{C}$, and the temperature maintained $18-22^{\circ} \mathrm{C}$ during the circulation arrest period. Whether to perform an aortic valve replacement depended on the condition of the aortic valve.
Total arch replacement (TAR) method was selected when major dissection teared around aortic arch or proximal descending aorta and/or diameter of aortic arch was greater than $4 \mathrm{~cm}$ on contrast CTA. Systemic rewarming was started after the completion of anastomosis to the left common carotid artery and the proximal reconstruction marked the completion of a standard CPB surgery. All patients were then transferred to ICU for routine monitoring.

\section{Statistical analysis}

Categorical variables were presented as frequencies with percentages and analyzed with Chi-square or Fisher exact test, as appropriate. Continuous variables were expressed as median and interquartile (IQR). The student $t$-test was applied for normally distributed continuous variables and the Mann-Whitney $U$ test for nonnormally distributed variables.

To identify potential risk factors for the development of AKI, multivariable logistic regression analyses were performed. Considering the relatively low number of AKI $(n=114)$ in the cohort and in order to avoid overfitting in the model, variables with $\mathrm{P}<0.50$ were selected for further multivariable logistic regression models. 12 parameters were identified (Table 3). Kaplan-Meier survival curve was applied for long-term survival analysis which further examined by 
Table 3 Multivariable analysis of risk factors for acute kidney injury with KDIGO

\begin{tabular}{lccc}
\hline Variable & OR & $95 \% \mathrm{Cl}$ & $\mathrm{P}$ value \\
\hline Age & 1.031 & $0.951-1.119$ & 0.456 \\
Previous cardiac surgery & 1.143 & $0.130-10.063$ & 0.904 \\
$\mathrm{sCr}$ & 0.994 & $0.983-1.004$ & 0.219 \\
PLT & 0.999 & $0.990-1.008$ & 0.790 \\
Fibrinogen & 1.069 & $0.717-1.595$ & 0.743 \\
Left renal artery: true lumen & 0.410 & $0.113-1.493$ & 0.176 \\
Right renal artery: true lumen & 0.691 & $0.172-2.766$ & 0.601 \\
CPB time & 1.003 & $0.991-1.015$ & 0.640 \\
PPC time & 1.029 & $0.999-1.060$ & 0.058 \\
DHCA time & 0.983 & $0.933-1.036$ & 0.522 \\
Drainage volume 24 hours after & 1.001 & $1.000-1.002$ & 0.248 \\
surgery & & & \\
Ventilation time (hour) & 1.029 & $1.007-1.051$ & 0.009 \\
\hline
\end{tabular}

SCr, serum creatinine; PLT, platelet; CPB, cardiopulmonary bypass; PPC, posterior parallel circulation; DHCA, deep hypothermic circulatory arrest; $\mathrm{OR}$, odds ratio; $\mathrm{Cl}$, confidence interval. $\mathrm{P}<0.05$ was considered statistically significant.

\section{Log-Rank test.}

All analyses were two-sided, and $\mathrm{P}<0.05$ was considered statistically significant. Data was analyzed with SPSS software for windows (version 25.0 IBM Corp, Armonk, NY).

\section{Results}

\section{Demographic and clinical features}

A total of 214 patients who received TA-AAD emergency operation were enrolled in this retrospective study and divided into AKI group (114 cases) and non-AKI group (100 cases). Patients' preoperative characteristics were presented in Table 4. According to KIDGO criteria, 114 (53.3\%) patients were diagnosed with postoperative AKI and 43 (20.1\%) required RRT. The median age of patients with or without AKI was $68.0(64.0,74.0)$ and $66.0(62.0,72.8)$ years respectively. Our data suggested that there was no significant difference identified in preoperative parameters and operative variables between two groups (Tables 4,5).

\section{Short-term outcomes}

As indicated in Table 2, patients in AKI group had a significantly increased incidence of having in-hospital complications compared to non-AKI group. The percentage of patients required dialysis treatment was significantly higher in AKI group (27.2\%) compared to non-AKI group $(12.0 \%)(\mathrm{P}=0.006)$. In addition, we discovered that patients complicated with AKI required longer mechanical ventilation, ICU and total hospitalization stay. The 30 -day mortality rate was $21.5 \%$ in all enrolled patients with $26.3 \%$ in AKI group and $16.0 \%$ in non-AKI group $(\mathrm{P}=0.067)$ respectively. Longer mechanical ventilation duration $(\mathrm{OR}$, 1.029 ; 95\% CI, $1.007-1.051 ; \mathrm{P}=0.009$ ) was identified as an independent risk factor associated with postoperative AKI based on the multivariable analysis (Table 3).

\section{Long-term outcome}

34 patients in AKI group and 16 in non-AKI group died during hospitalization and were excluded from the longterm outcome analysis. As a result, a total of 80 AKI patients and 84 non-AKI were included in the follow-up. 10 patients were lost during follow-up period, including 4 patients in AKI group and 6 in non-AKI group, and they were excluded from the subsequent long-term survival analysis. The median follow-up period was 29 months. We identified a total of 14 deaths in the follow-up period, including 11 patients in AKI group and 3 in non-AKI group. The most frequent cause of late mortality was aortic rupture, followed by cardiac failure and respiratory failure (Table 6). The Kaplan-Meier test suggested a significant difference of long-term survival rate between patients with and without AKI $(\mathrm{P}=0.019)$ (Figure 1).

\section{Discussion}

In this retrospective study, we identified the incidence, risk factors, short-term and long-term outcomes for elderly TA-AAD patients who complicated with AKI. Among all enrolled 214 patients, 114 (53.3\%) developed postoperative AKI and $43(20.1 \%)$ of whom required RRT. The 30 -day mortality rate was $26.3 \%$ in AKI group and $16.0 \%$ in non-AKI group. Multivariable logistic regression analysis demonstrated that longer mechanical ventilation duration was an independent risk factor for developing postoperative AKI. Our data suggested that shorten mechanical ventilation duration as much as possible might help reducing postoperative AKI incidence. To our knowledge, this was the first study to examine the incidence, risk factors and long-term outcomes for AKI complication after TA- 
Table 4 Comparison of preoperative variables

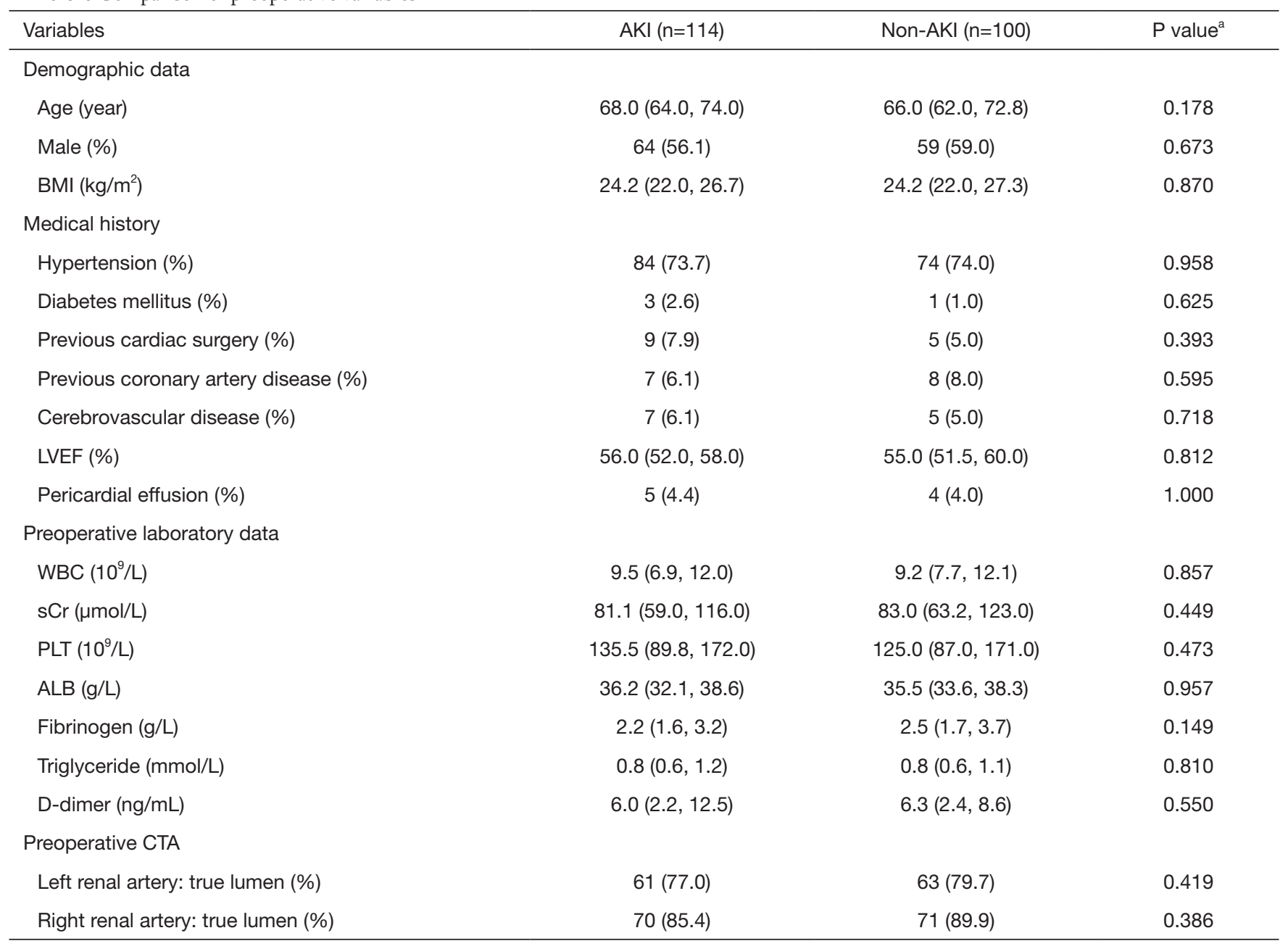

Data presented as n (\%); median (IQR). BMI, body mass index; LVEF, left ventricular ejection fraction; WBC, white blood cell; SCr, serum creatinine; PLT, platelet; ALB, albumin; CTA, computed tomography angiography. ${ }^{\mathrm{P}} \mathrm{P}$ values indicate differences between AKI and Non-AKI. $\mathrm{P}<0.05$ was considered statistically significant.

Table 5 Comparison of operative variables

\begin{tabular}{lccc}
\hline Variables & AKI $(\mathrm{n}=114)$ & Non-AKI $(\mathrm{n}=100)$ & $\mathrm{P}_{\text {value }} \mathrm{a}^{\mathrm{a}}$ \\
\hline TAR $(\%)$ & $53(46.5)$ & $35(35.0)$ & 0.088 \\
CABG/MVR/MVP/TVP (\%) & $12(10.5)$ & $25(25.0)$ & 0.899 \\
Aortic valve (\%) & $28(24.6)$ & $213.5(179.5,252.0)$ & 0.941 \\
CPB time (min) & $225.0(187.5,276.5)$ & $43.5(32.3,58.0)$ & 0.178 \\
PPC time (min) & $48.0(35.0,68.0)$ & $154.0(123.0,190.0)$ & 0.177 \\
Aortic cross-clamp time (min) & $153.0(120.0,189.5)$ & $25.5(16.3,35.8)$ & 0.939 \\
DHCA time (min) & $28.0(19.0,35.0)$ & 0.359 \\
\hline
\end{tabular}

Data presented as n (\%); median (IQR). TAR, total arch replacement; CABG, coronary artery bypass graft; MVR, mitral valve replacement; MVP, mitral valvuloplasty; TVP, tricuspid valvuloplasty; CPB, cardiopulmonary bypass; PPC, posterior parallel circulation; DHCA, deep hypothermic circulatory arrest. ${ }^{a} \mathrm{P}$ values indicate differences between $\mathrm{AKI}$ and non-AKI. $\mathrm{P}<0.05$ was considered statistically significant. 
Table 6 Causes of late mortality $(n=14)$

\begin{tabular}{lcc}
\hline \multirow{2}{*}{ Cause of late mortality } & \multicolumn{2}{c}{ Overall cohort } \\
\cline { 2 - 3 } & AKI $(\mathrm{n}=11)$ & Non-AKI $(\mathrm{n}=3)$ \\
\hline Aortic rupture & 4 & 1 \\
Cardiac failure & 2 & 1 \\
Respiratory failure & 1 & 1 \\
Unknown & 4 & 1 \\
\hline
\end{tabular}



Figure 1 Kaplan-Meier estimate for late survival of patients operated on for type A acute aortic dissection according to the presence of postoperative acute kidney injury (AKI).

AAD emergency surgery in elderly patients.

$\mathrm{AKI}$ is an important complication after cardiac surgery and closely associates with worse prognosis (7). While there are multiple published studies on incidence of postoperative AKI, no consensus has been reached yet. Sasabuchi and his colleagues (3) reported that the incidence of AKI in aortic arch surgery was $44.9 \%$ and only 14 cases needed RRT, which was lower than what we reported in this study. The higher incidence of AKI $(53.3 \%)$ in this study indicated that the elderly patients might more vulnerable to kidney injury after surgery, as suggested in previous studies that advanced age being an important risk factor for postoperative AKI (8-10).

The overall 30 -day mortality was $21.5 \%$ (46 of 214 patients), which seemed higher than previous reported $(1,3,11)$. However, this result proved the idea that advanced age being an important risk for increased morbidity and mortality rate in TA-AAD patients after surgery
(12-15). This age-related difference may be explained by that elderly patients are prone to develop lethal multiple organs dysfunction syndrome (MODS) when complicating with AKI (16). Several studies have suggested that postoperative AKI was associated with increased mortality in aortic surgery. However, we only observed a trend but did not reach statistical significance in 30-day mortality rate between groups in this study. This may due to the relatively small number of patients we enrolled in this study. However, we did discover a significant difference in the long-term mortality rate between patients with and without postoperative AKI. A previous meta-analysis shown that patients with AKI had higher risk of developing chronic kidney disease and end-stage renal dysfunction (17). Although renal function after hospital discharge was not evaluated in the present study, it is only reasonable to speculate that patients with AKI were more likely to have chronic kidney disease and/or end-stage renal disease and contributed to their higher mortality rate. As suggested in this study, early discovering patients with increased risk and preventing postoperative AKI are crucial to improve elderly patients' prognosis.

Longer mechanical ventilation duration was identified as the only independent risk factor for postoperative AKI base on the logistic regression model, which was consistent with previous study that the prolonged usage of ventilator was an important trigger for AKI in patients underwent cardiac surgeries (18). Van den Akker and colleagues suggested in their meta-analysis that mechanical ventilation longer than 24 hours was associated with a threefold increase in the risk for developing AKI in critically ill patients (19). Even though it has not been well established, the prolonged mechanical ventilation related AKI and even acute renal failure (ARF) may be explained by three possible mechanisms: (I) through effects on arterial blood gases; (II) through an effect on systemic and renal blood flow; (III) by triggering a pulmonary inflammatory reaction induced during biotrauma that further mediates systemic changes (20). Based on what we have learnt from this study, we recommend to minimize the mechanical ventilation duration as much as possible in order to reduce the risk of developing AKI after surgeries.

Multiple factors might affect postoperative respiratory status following TA-AAD surgery and prolonged mechanical ventilation duration. Li et al. reported that increased leukocytes, age and time from symptom onset to surgery were risk factors for prolonged mechanical ventilation for TA-AAD patients (21). Kimura and colleagues found that 
preoperative shock history, limb ischemia and concomitant coronary artery bypass grafting (CABG) increased the risk of prolonged mechanical ventilation after TA-AAD surgery (22). As prolonged mechanical ventilation was identified as a risk factor of postoperative AKI for elderly TA-AAD patients in our study, acknowledging potential confounding factors that might prolong mechanical ventilation might benefit patients' postoperative management.

In contrast with previous studies $(1,23)$, we found increased preoperative $\mathrm{sCr}$ level was not associated with worse renal function in our cohort which might be explained by following reasons. SCr is not always a perfect surrogate of renal function, for its dependence on several nonrenal factors (24). An increase of creatinine may be measured only after a considerable loss of glomerular filtration rate (GFR) and has a delayed effect of reflecting acute renal damage. Novel biomarkers, such as neutrophil gelatinase-associated lipocalin (NGAL) and cystatin $\mathrm{C}$, have been correlated with the duration and severity of AKI after adult cardiac surgery and have been identified as better independent predictors of AKI, when compared to conventional biomarkers (25). Similarly, a previous study suggested that measurement of sCr on arrival at ICU after cardiac surgery was not a reliable predictor of AKI (26). Therefore, studies were needed to explore the value of novel biomarkers in predicting duration and severity of postoperative AKI in elderly TA-AAD patients.

Forty-three (20.1\%) AKI patients in this study required postoperative RRT. This percentage was much higher compared to other studies which ranging from $6 \%$ to $14 \%(1,3,11,14,15)$. Roh and colleagues (1) suggested that prompt application of RRT might improve outcomes. However, as presented in our previous study, we discovered that patients with timely postoperative RRT still suffered from higher perioperative mortality and postoperative morbidity rates (27). RRT procedure itself may lead to circulation instability, infection, thrombosis, electrolyte imbalance and other complications that may affect patients' recovery and prognosis (28). This study demonstrated that a higher incidence of AKI was associated with a higher dialysis rate and a worse long-term mortality rate.

\section{Study limitations}

This study has its limitations. Firstly, this was a singlecenter retrospective study, with a cohort that might not necessarily representable for the general population. Secondly, many previous studies $(1,14,23,29)$ used RIFLE or AKIN classification as the diagnostic criteria for AKI while we used KDIGO in the current study. This difference may induce some difficulties when comparing our results with previous studies. Thirdly, although the causes of deaths after discharge were collected in this study, but they were not confirmed by autopsy. Finally, we only used the changes in sCr level for KDIGO classification since accurate data regarding urine output was unavailable which might result in an underestimation of the incidence of AKI.

\section{Conclusions}

In conclusion, our study demonstrated that the development of AKI after TA-AAD surgery was common in elderly patients and it adversely affected long-term survival. Prolonged postoperative mechanical ventilation duration was associated with the development of AKI. To improve the prognosis in elderly patients who received TAAAD surgery, more attention should be paid to prevent postoperative AKI.

\section{Acknowledgments}

Funding: None.

\section{Footnote}

Reporting Checklist: The authors have completed the STROBE reporting checklist. Available at http://dx.doi. org/10.21037/jtd-20-2018

Data Sharing Statement: Available at http://dx.doi. org/10.21037/jtd-20-2018

Conflicts of Interest: All authors have completed the ICMJE uniform disclosure form (available at http://dx.doi. org/10.21037/jtd-20-2018). The authors have no conflicts of interest to declare.

Ethical Statement: The authors are accountable for all aspects of the work in ensuring that questions related to the accuracy or integrity of any part of the work are appropriately investigated and resolved. The ethics committee of Nanjing Drum Tower hospital approved this retrospective study and waived the need for individual informed consent due to the nature of this study. The study was conducted in accordance with the Declaration of Helsinki (as revised in 2013). 
Open Access Statement: This is an Open Access article distributed in accordance with the Creative Commons Attribution-NonCommercial-NoDerivs 4.0 International License (CC BY-NC-ND 4.0), which permits the noncommercial replication and distribution of the article with the strict proviso that no changes or edits are made and the original work is properly cited (including links to both the formal publication through the relevant DOI and the license). See: https://creativecommons.org/licenses/by-nc-nd/4.0/.

\section{References}

1. Roh GU, Lee JW, Nam SB, et al. Incidence and risk factors of acute kidney injury after thoracic aortic surgery for acute dissection. Ann Thorac Surg 2012;94:766-71.

2. Suri R. Acute Kidney Injury Is Associated With Increased Long-Term Mortality After Cardiothoracic Surgery. Yearbook of Cardiology 2010;2010:209-11.

3. Sasabuchi Y, Kimura N, Shiotsuka J, et al. Long-Term Survival in Patients With Acute Kidney Injury After Acute Type A Aortic Dissection Repair. Ann Thorac Surg 2016;102:2003-9.

4. Garrick R. Acute Kidney Injury Increases Risk of ESRD among Elderly. Yearbook of Medicine 2009;2009:266-8.

5. Kidney Disease: Improving Global Outcomes(KDIGO) Acute Kidney Injury Work Group. KDIGO Clinical Practice Guideline for Acute Kidney Injury. Kidney inter 2012;2:1-138.

6. Joannidis M, Druml W, Forni LG, et al. Prevention of acute kidney injury and protection of renal function in the intensive care unit: update 2017 : Expert opinion of the Working Group on Prevention, AKI section, European Society of Intensive Care Medicine. Intensive Care Med 2017;43:730-49.

7. Lingzhi C, Hao Z, Weijian H, et al. Outcome Predictors in Patients Presenting With Acute Aortic Dissection. J Cardiothorac Vasc Anesth 2016;30:1272-7.

8. Wang J, Yu W, Zhai G, et al. Independent risk factors for postoperative AKI and the impact of the AKI on 30-day postoperative outcomes in patients with type A acute aortic dissection: an updated meta-analysis and meta-regression. J Thorac Dis 2018;10:2590-8.

9. Amano K, Takami Y, Ishikawa H, et al. Lower body ischaemic time is a risk factor for acute kidney injury after surgery for type A acute aortic dissection. Interact Cardiovasc Thorac Surg 2020;30:107-12.

10. Fang $Z$, Wang G, Liu Q, et al. Moderate and deep hypothermic circulatory arrest has a comparable effect on acute kidney injury after total arch replacement with frozen elephant trunk procedure in type A aortic dissection. Interact Cardiovasc Thorac Surg 2019;29:130-6.

11. Ko T, Higashitani M, Sato A, et al. Impact of Acute Kidney Injury on Early to Long-Term Outcomes in Patients Who Underwent Surgery for Type A Acute Aortic Dissection. Am J Cardiol 2015;116:463-8.

12. Conzelmann LO, Weigang E, Mehlhorn U, et al. Mortality in patients with acute aortic dissection type A: analysis of pre- and intraoperative risk factors from the German Registry for Acute Aortic Dissection Type A (GERAADA). Eur J Cardiothorac Surg 2016;49:e44-52.

13. Rylski B, Suedkamp M, Beyersdorf F, et al. Outcome after surgery for acute aortic dissection type A in patients over 70 years: data analysis from the German Registry for Acute Aortic Dissection Type A (GERAADA). Eur J Cardiothorac Surg 2011;40:435-40.

14. Tsai HS, Tsai FC, Chen YC, et al. Impact of acute kidney injury on one-year survival after surgery for aortic dissection. Ann Thorac Surg 2012;94:1407-12.

15. Schoenrath F, Laber R, Maralushaj M, et al. Survival, Neurologic Injury, and Kidney Function after Surgery for Acute Type A Aortic Dissection. Thorac Cardiovasc Surg 2016;64:100-7.

16. Chronopoulos A, Rosner MH, Cruz DN, et al. Acute kidney injury in elderly intensive care patients: a review. Intensive Care Med 2010;36:1454-64.

17. Coca SG, Singanamala S, Parikh CR. Chronic kidney disease after acute kidney injury: a systematic review and meta-analysis. Kidney Int 2012;81:442-8.

18. Heringlake M, Nowak Y, Schon J, et al. Postoperative intubation time is associated with acute kidney injury in cardiac surgical patients. Crit Care 2014;18:547.

19. van den Akker JP, Egal M, Groeneveld AB. Invasive mechanical ventilation as a risk factor for acute kidney injury in the critically ill: a systematic review and metaanalysis. Crit Care 2013;17:R98.

20. Kuiper JW, Groeneveld AB, Slutsky AS, et al. Mechanical ventilation and acute renal failure. Crit Care Med 2005;33:1408-15.

21. Li CN, Chen L, Ge YP, et al. Risk factors for prolonged mechanical ventilation after total aortic arch replacement for acute DeBakey type I aortic dissection. Heart Lung Circ 2014;23:869-74.

22. Kimura N, Tanaka M, Kawahito K, et al. Risk factors for prolonged mechanical ventilation following surgery for acute type a aortic dissection. Circ J 2008;72:1751-7.

23. Zhao H, Pan X, Gong Z, et al. Risk factors for acute 
kidney injury in overweight patients with acute type A aortic dissection: a retrospective study. J Thorac Dis 2015;7:1385-90.

24. Herget-Rosenthal S, Marggraf G, Husing J, et al. Early detection of acute renal failure by serum cystatin $\mathrm{C}$. Kidney Int 2004;66:1115-22.

25. Haase M, Bellomo R, Devarajan P, et al. Novel biomarkers early predict the severity of acute kidney injury after cardiac surgery in adults. Ann Thorac Surg 2009;88:124-30.

26. Haase-Fielitz A, Bellomo R, Devarajan P, et al. Novel and conventional serum biomarkers predicting acute kidney injury in adult cardiac surgery--a prospective cohort study.
Crit Care Med 2009;37:553-60.

27. Wang Z, Ge M, Chen T, et al. Independent risk factors and the long-term outcomes for postoperative continuous renal replacement treatment in patients who underwent emergency surgery for type a acute aortic dissection. J Cardiothorac Surg 2020;15:100.

28. Hanafusa N, Nakai S, Iseki K, et al. Japanese society for dialysis therapy renal data registry-a window through which we can view the details of Japanese dialysis population. Kidney Int Suppl (2011) 2015;5:15-22.

29. Wu HB, Qin H, Ma WG, et al. Can Renal Resistive Index Predict Acute Kidney Injury After Acute Type A Aortic Dissection Repair? Ann Thorac Surg 2017;104:1583-9.

Cite this article as: Wang Z, Ge M, Chen T, Chen C, Zong Q, Lu L, Li K, Wang D. Risk factors and long-term outcomes of elderly patients complicating with acute kidney injury after type A acute aortic dissection surgery: a retrospective study. J Thorac Dis 2020;12(10):5833-5841. doi: 10.21037/jtd-20-2018 